TITLE:

\title{
Computational multiple scattering analysis of elastic waves in unidirectional composites
}

$\operatorname{AUTHOR}(S)$ :

Sumiya, Takuto; Biwa, Shiro; Haïat, Guillaume

\section{CITATION:}

Sumiya, Takuto ...[et al]. Computational multiple scattering analysis of elastic waves in unidirectional composites. Wave Motion 2013, 50(2):

253-270

ISSUE DATE:

2013-03

URL:

http://hdl.handle.net/2433/170208

\section{RIGHT:}

(c) 2012 Elsevier B.V.; This is not the published version. Please cite only the published version.; この論文は出版社版でありません。引用の際に は出版社版をご確認ご利用ください。 
This article appeared as:

T. Sumiya, S. Biwa and G. Haïat, Computational multiple scattering analysis of elastic waves in unidirectional composites, Wave Motion, 50 (2013), pp. 253-270.

\title{
Computational multiple scattering analysis of elastic waves in unidirectional composites
}

\author{
Takuto Sumiya ${ }^{\mathrm{a}}$, Shiro Biwa ${ }^{\mathrm{b}^{*}}$ and Guillaume Haïat ${ }^{\mathrm{c}}$ \\ ${ }^{a}$ Department of Micro Engineering, Graduate School of Engineering, Kyoto University, \\ Yoshida-honmachi, Sakyo-ku, Kyoto 606-8501, Japan \\ ${ }^{b}$ Department of Aeronautics and Astronautics, Graduate School of Engineering, Kyoto \\ University, Yoshida-honmachi, Sakyo-ku, Kyoto 606-8501, Japan \\ ${ }^{c}$ CNRS, Université Paris-Est, Laboratoire de Modélisation et de Simulation Multi-Echelle, \\ UMR CNRS 8208, 61 avenue du Général de Gaulle, 94010 Créteil cedex, France
}

\begin{abstract}
A numerical procedure is presented in this paper for the two-dimensional, time-harmonic elastodynamic multiple scattering problems for unidirectional fiber-reinforced composites. The proposed procedure is based on the eigenfunction expansion of the displacement potentials and the numerical collocation method to solve the expansion coefficients, and is capable of modeling arbitrary fiber arrangements. To demonstrate the applicability of the procedure, the $\mathrm{P}$ and SV wave propagation characteristics in unidirectional fiber-reinforced composites are analyzed for different fiber arrangements and fiber volume fractions. The simulated results are shown to capture the detailed features of the local wave fields in the composites accompanying the mode conversion. From the computed wave fields, the effective phase velocities of the composites are identified as functions of the frequency, and found to be in good agreement with the predictions of a micromechanical model for random composites. The energy transmission spectra of the $\mathrm{P}$ and SV waves are also demonstrated, which exhibit the stop-band formation for the composites with regular fiber arrangements.
\end{abstract}

Keywords: Elastic wave; Fiber-reinforced composite material; Multiple scattering; Stop band formation; Effective phase velocity

\footnotetext{
* Corresponding author: Shiro Biwa, Department of Aeronautics and Astronautics, Graduate School of Engineering, Kyoto University. E-mail: biwa@kuaero.kyoto-u.ac.jp
} 


\section{Introduction}

Fiber-reinforced composite materials have found increasing application in a variety of areas such as aerospace engineering owing to their enhanced mechanical properties. Understanding of the elastic wave propagation characteristics in these materials is important regarding the design against dynamic loadings as well as the nondestructive characterization using ultrasonic waves. Elastic waves in fiber-reinforced composites undergo multiple scattering by their microstructures and exhibit frequency-dependent propagation characteristics $[1,2]$. In the nondestructive testing, these features need to be clarified in order to fully interpret the acquired ultrasonic waveforms and to obtain information of e.g. the fiber-matrix interfacial properties [3].

Elastic wave propagation in fiber-reinforced composites has been studied extensively, based on different versions of multiple scattering theories and micromechanical models. In multiple scattering theories for fiber-reinforced composite media [4-8], the infinite hierarchy of the governing equations is truncated by introducing certain assumptions for the spatial correlation of fiber positions to obtain the average wave field and the effective wave numbers. Other existing models assume either ideally periodic microstructures as approached by the Bloch (or Floquet) theory and unit-cell analysis [9-12], or random microstructures as approached by the effective medium theories and other micromechanical models [13-19]. Actual fiber-reinforced composites, however, often show intermediate microstructural features. For example, some metal-matrix composites are manufactured by laying up and hot-pressing matrix/fiber mats (so-called monotapes), which contain a single row of continuous fibers [20, 21]. Such a process results in internal fiber arrangements which are fairly regular and periodic but not perfectly so, since the number of the stacked monotapes is finite and certain disorder of fiber positioning occurs.

In order to investigate the elastic wave propagation characteristics in composites with arbitrary fiber arrangements, it is desirable to consider more direct simulation approaches. In this regard, Cai and Williams [22-24] proposed a numerical technique called scatterer polymerization for large-scale multiple scattering problems of scalar shear waves in fiber-reinforced composites. Biwa et al. [25] presented a semi-analytical procedure to solve the equations of time-harmonic shear-wave multiple scattering directly, based on the eigenfunction expansion of the wave field and numerical collocation technique to solve the expansion coefficients. Using this method, the interaction phenomena of shear waves with different arrangements of fibers have been analyzed effectively, including the stop-band formation in regular fiber arrangements and its dependence on their finite length [26] as well as the influence of the disordered fiber arrangement on the transmission characteristics [27]. Furthermore, the effective shear-wave phase velocity and attenuation coefficient of a fiber-reinforced composite obtained by the simulations have been compared favorably with experimental results [28].

The above-mentioned foregoing works, however, deal with the shear waves polarized parallel to the aligned fibers, which are commonly termed as SH (shear horizontal) waves. In this situation, no mode conversion occurs at scattering, and the equations to be solved are of a scalar nature. Consequently, it remains as an intriguing task to examine more general problems of two-dimensional elastic waves interacting with unidirectional fibers, for which mode conversion phenomena are relevant. In this paper, the above-mentioned numerical procedure [25] is extended to the more general setting of two-dimensional elastodynamics, in order to analyze multiple scattering of elastic waves in unidirectional fiber-reinforced 
composites. Although two-dimensional multiple scattering problems for fiber-reinforced composites have been approached by the abovementioned mathematical treatments as well as by different numerical methods [29-31], the semi-analytical eigenfunction expansion approach is worth thorough exploitation for its effectiveness and high accuracy. To demonstrate the application of the proposed procedure, the multiple scattering simulations are presented for elastic waves in unidirectional composites with different fiber arrangements, and the effective phase velocities and the energy transmission behavior are illustrated for a specific type of composites.

\section{Formulation}

\subsection{Governing equations}

The problem considered in this paper consists of the two-dimensional multiple scattering of elastic waves in an infinite elastic medium (Lamé constants $\lambda_{1}, \mu_{1}$ and density $\rho_{1}$ ) containing $N$ circular cylindrical elastic fibers (radius $a$, Lamé constants $\lambda_{2}, \mu_{2}$ and density $\rho_{2}$ ) which are all aligned parallel to the $x_{3}$-axis and arranged arbitrarily in the $x_{1}-x_{2}$ plane, see Fig. 1 . Below, the position vector of a generic point is denoted by $\mathbf{r}$, and the position vector of the center of the ith fiber by $\mathbf{r}_{i}(i=1,2, \ldots \ldots, N)$. The domains occupied by the matrix and the fibers are denoted as $D_{1}$ and $D_{2}$, respectively.

For the time-harmonic problems with time-dependence of the form exp(-i $\omega t)$ implicitly understood ( $\omega$ : angular frequency, $i=\sqrt{-1}$ ), the governing equations of the two-dimensional elastodynamics are written in terms of the displacement vector $\mathbf{u}$ by

$$
\mu_{\alpha} \nabla^{2} \mathbf{u}+\left(\lambda_{\alpha}+\mu_{\alpha}\right) \nabla(\nabla \cdot \mathbf{u})+\rho_{\alpha} \omega^{2} \mathbf{u}=\mathbf{0}, \quad \text { in } D_{\alpha}(\alpha=1,2),
$$

where $\nabla$ is the two-dimensional gradient operator, and the subscript $\alpha$ corresponds to the matrix ( $\alpha=1)$ or the fiber $(\alpha=2)$. The Helmholtz theorem allows the two-dimensional displacement fields $u_{1}(\mathbf{r})$ and $u_{2}(\mathbf{r})$ to be derived from two potential functions $\Phi(\mathbf{r})$ and $\Psi(\mathbf{r})$ as [32]

$$
u_{1}(\mathbf{r})=\frac{\partial \Phi}{\partial x_{1}}+\frac{\partial \Psi}{\partial x_{2}}, u_{2}(\mathbf{r})=\frac{\partial \Phi}{\partial x_{2}}-\frac{\partial \Psi}{\partial x_{1}},
$$

where $\Psi$ is the $x_{3}$-component of the vector potential. For the displacements and potentials, subscripts to identify the pertinent domains $\left(D_{1}\right.$ or $\left.D_{2}\right)$ are omitted as it is evident in the context. These potentials satisfy the following Helmholtz equations,

$$
\nabla^{2} \Phi+k_{\mathrm{L} \alpha}{ }^{2} \Phi=0, \quad \nabla^{2} \Psi+k_{\mathrm{T} \alpha}{ }^{2} \Psi=0, \quad \text { in } D_{\alpha}(\alpha=1,2),
$$

where the wave numbers and the pertinent wave speeds are given by

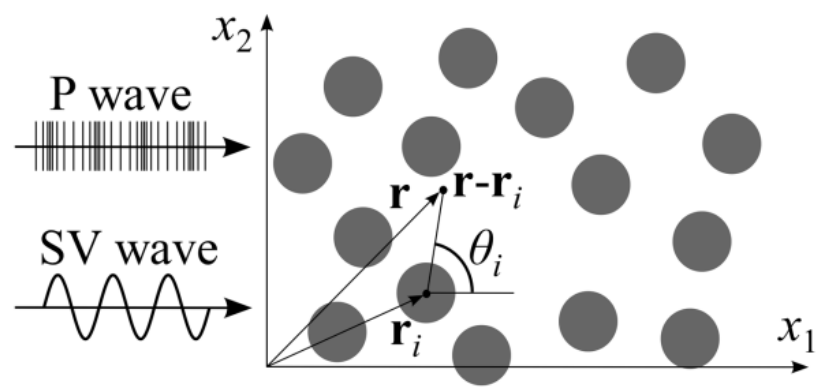

Fig. 1 Schematic representation of a unidirectional composite. 


$$
k_{\mathrm{L} \alpha}=\frac{\omega}{c_{\mathrm{L} \alpha}}, \quad k_{\mathrm{T} \alpha}=\frac{\omega}{c_{\mathrm{T} \alpha}}, \quad c_{\mathrm{L} \alpha}=\sqrt{\frac{\lambda_{\alpha}+2 \mu_{\alpha}}{\rho_{\alpha}}}, \quad c_{\mathrm{T} \alpha}=\sqrt{\frac{\mu_{\alpha}}{\rho_{\alpha}}} \quad(\alpha=1,2) .
$$

The incident wave is assumed to be a plane wave propagating in the positive $x_{1}$-direction, either with longitudinal or transverse polarization. The displacement potentials of the incident wave are given by

$$
\Phi^{\mathrm{inc}}(\mathbf{r})=\Phi_{0} \exp \left(\mathrm{i} k_{\mathrm{L} 1} x_{1}\right), \quad \Psi^{\mathrm{inc}}(\mathbf{r})=0,
$$

for the longitudinal wave incidence, and

$$
\Phi^{\mathrm{inc}}(\mathbf{r})=0, \Psi^{\mathrm{inc}}(\mathbf{r})=\Psi_{0} \exp \left(\mathrm{i}_{\mathrm{T} 1} x_{1}\right),
$$

for the transverse wave incidence, where $\Phi_{0}$ and $\Psi_{0}$ are constants. The following formulation applies to these two kinds of incident waves in a unified manner. Hereafter, the longitudinal and transverse waves are also referred to as the P (compressional) and SV (shear vertical) waves, respectively, according to the common terminology.

The displacement potentials in the matrix are expressed by the sum of the incident and the scattered fields as

$$
\Phi(\mathbf{r})=\Phi^{\mathrm{inc}}(\mathbf{r})+\sum_{i=1}^{N} \Phi^{i, \mathrm{sca}}(\mathbf{r}), \Psi(\mathbf{r})=\Psi^{\mathrm{inc}}(\mathbf{r})+\sum_{i=1}^{N} \Psi^{i, \mathrm{sca}}(\mathbf{r}),
$$

where $\Phi^{i, \text { sca }}(\mathbf{r})$ and $\Psi^{i, \text { sca }}(\mathbf{r})$ are the potentials of the scattered wave from the ith fiber. The scattered wave potentials also satisfy Helmholtz equations together with the radiation condition at infinity, and can be expressed as

$$
\Phi^{i, \text { sca }}(\mathbf{r})=\sum_{n=-\infty}^{\infty} A_{n}{ }^{i} H_{n}\left(k_{\mathrm{L} 1}\left|\mathbf{r}-\mathbf{r}_{i}\right|\right) \exp \left(\mathrm{in} \theta_{i}\right), \quad \Psi^{i, \text { sca }}(\mathbf{r})=\sum_{n=-\infty}^{\infty} B_{n}{ }^{i} H_{n}\left(k_{\mathrm{T} 1}\left|\mathbf{r}-\mathbf{r}_{i}\right|\right) \exp \left(\mathrm{in} \theta_{i}\right),
$$

where $A_{n}{ }^{i}$ and $B_{n}{ }^{i}(n=0, \pm 1, \pm 2, \ldots ; \quad i=1,2, \ldots, N)$ are unknown coefficients, and $\theta_{i}$ is the polar angle of $\mathbf{r}$ viewed from the position $\mathbf{r}_{\boldsymbol{i}}$, c.f. Fig. 1 .

The $i$ th fiber generates the scattered waves in response to the wave fields which impinge on that fiber. The latter are called exciting fields, and are given by the sum of the incident wave and the scattered waves from all the other fibers, namely,

$$
\Phi^{i, \text { exc }}(\mathbf{r})=\Phi^{\mathrm{inc}}(\mathbf{r})+\sum_{\substack{j=1 \\ j \neq i}}^{N} \Phi^{j, \text { sca }}(\mathbf{r}), \quad \Psi^{i, \text { exc }}(\mathbf{r})=\Psi^{\mathrm{inc}}(\mathbf{r})+\sum_{\substack{j=1 \\ j \neq i}}^{N} \Psi^{j, \text { sca }}(\mathbf{r}) .
$$

The exciting waves are also expressed as solutions of Helmholtz equation which are regular at $\mathbf{r}_{i}$, i.e.,

$$
\Phi^{i, \operatorname{exc}}(\mathbf{r})=\sum_{n=-\infty}^{\infty} E_{n}{ }^{i} J_{n}\left(k_{\mathrm{L} 1}\left|\mathbf{r}-\mathbf{r}_{i}\right|\right) \exp \left(\mathrm{in} \theta_{i}\right), \quad \Psi^{i, \text { exc }}(\mathbf{r})=\sum_{n=-\infty}^{\infty} F_{n}{ }^{i} J_{n}\left(k_{\mathrm{T} 1}\left|\mathbf{r}-\mathbf{r}_{i}\right|\right) \exp \left(\mathrm{in} \theta_{i}\right),
$$

where $E_{n}{ }^{i}$ and $F_{n}{ }^{i}(n=0, \pm 1, \pm 2, \ldots ; \quad i=1,2, \ldots, N)$ are unknown coefficients.

In each fiber, there exist refracted wave fields which are expressed similarly by

$$
\Phi^{i, \text { ref }}(\mathbf{r})=\sum_{n=-\infty}^{\infty} C_{n}{ }^{i} J_{n}\left(k_{\mathrm{L} 2}\left|\mathbf{r}-\mathbf{r}_{i}\right|\right) \exp \left(\mathrm{in} \theta_{i}\right), \quad \Psi^{i, \text { ref }}(\mathbf{r})=\sum_{n=-\infty}^{\infty} D_{n}{ }^{i} J_{n}\left(k_{\mathrm{T} 2}\left|\mathbf{r}-\mathbf{r}_{i}\right|\right) \exp \left(\mathrm{in} \theta_{i}\right),
$$

where $C_{n}{ }^{i}$ and $D_{n}{ }^{i}(n=0, \pm 1, \pm 2, \ldots ; \quad i=1,2, \ldots, N)$ are also unknown coefficients.

The relations between the expansion coefficients $A_{n}{ }^{i}, B_{n}{ }^{i}, C_{n}{ }^{i}, D_{n}{ }^{i}, E_{n}{ }^{i}$ and $F_{n}{ }^{i}$ are established by appropriate boundary conditions at the matrix-fiber interfaces. In the present analysis, the matrix and the fibers are assumed to be perfectly bonded thus enforcing the continuity of the displacements and the 
tractions. The relations can be expressed by

$$
\left(\begin{array}{c}
A_{n}{ }^{i} \\
B_{n}{ }^{i} \\
C_{n}{ }^{i} \\
D_{n}{ }^{i}
\end{array}\right)=\left[\begin{array}{ll}
M_{11}^{(n)} & M_{12}^{(n)} \\
M_{21}^{(n)} & M_{22}^{(n)} \\
M_{31}^{(n)} & M_{32}^{(n)} \\
M_{41}^{(n)} & M_{42}^{(n)}
\end{array}\right]\left(\begin{array}{c}
E_{n}{ }^{i} \\
F_{n}{ }^{i}
\end{array}\right) .
$$

The elements of the matrix $\left[M^{(n)}\right]$ depend on the order $n$ but not on the position of the fiber. This matrix can be obtained by an ordinary procedure found in many classical references [33], and their elements are given explicitly in the Appendix. By writing

$$
S_{n}=M_{11}^{(n)}, \quad T_{n}=M_{12}^{(n)}, \quad U_{n}=M_{21}^{(n)}, \quad V_{n}=M_{22}^{(n)},
$$

the coefficients $A_{n}{ }^{i}$ and $B_{n}{ }^{i}$ are given in terms of $E_{n}{ }^{i}$ and $F_{n}{ }^{i}$ as

$$
A_{n}{ }^{i}=S_{n} E_{n}{ }^{i}+T_{n} F_{n}{ }^{i}, \quad B_{n}{ }^{i}=U_{n} E_{n}{ }^{i}+V_{n} F_{n}{ }^{i} .
$$

Equations (8), (10) and (14) are substituted into Eq. (9) to obtain the following equations which hold at arbitrary $\mathbf{r}$ in the matrix;

$$
\begin{aligned}
& \sum_{n=-\infty}^{\infty} E_{n}{ }^{i} J_{n}\left(k_{\mathrm{L} 1}\left|\mathbf{r}-\mathbf{r}_{i}\right|\right) \exp \left(\mathrm{in} \theta_{i}\right)=\Phi^{\mathrm{inc}}(\mathbf{r})+\sum_{\substack{j=1 \\
j \neq i}}^{N} \sum_{m=-\infty}^{\infty}\left(S_{m} E_{m}{ }^{j}+T_{m} F_{m}{ }^{j}\right) H_{m}\left(k_{\mathrm{L} 1}\left|\mathbf{r}-\mathbf{r}_{j}\right|\right) \exp \left(\mathrm{im} \theta_{j}\right) \\
& \sum_{n=-\infty}^{\infty} F_{n}{ }^{i} J_{n}\left(k_{\mathrm{T} 1}\left|\mathbf{r}-\mathbf{r}_{i}\right|\right) \exp \left(\mathrm{in} \theta_{i}\right)=\Psi^{\mathrm{inc}}(\mathbf{r})+\sum_{\substack{j=1 \\
j \neq i}}^{N} \sum_{m=-\infty}^{\infty}\left(U_{m} E_{m}{ }^{j}+V_{m} F_{m}{ }^{j}\right) H_{m}\left(k_{\mathrm{T} 1}\left|\mathbf{r}-\mathbf{r}_{j}\right|\right) \exp \left(\mathrm{i} m \theta_{j}\right) .
\end{aligned}
$$

In the above expressions, the $E_{n}{ }^{i}$ and $F_{n}{ }^{i}$ are unknown variables which should be determined numerically. If these coefficients are obtained, the potential fields are given by

$$
\begin{aligned}
& \Phi(\mathbf{r})=\Phi^{\mathrm{inc}}(\mathbf{r})+\sum_{j=1}^{N} \sum_{m=-\infty}^{\infty}\left(S_{m} E_{m}{ }^{j}+T_{m} F_{m}{ }^{j}\right) H_{m}\left(k_{\mathrm{L} 1}\left|\mathbf{r}-\mathbf{r}_{j}\right|\right) \exp \left(\mathrm{im} \theta_{j}\right), \\
& \Psi(\mathbf{r})=\Psi^{\mathrm{inc}}(\mathbf{r})+\sum_{j=1}^{N} \sum_{m=-\infty}^{\infty}\left(U_{m} E_{m}{ }^{j}+V_{m} F_{m}{ }^{j}\right) H_{m}\left(k_{\mathrm{T} 1}\left|\mathbf{r}-\mathbf{r}_{j}\right|\right) \exp \left(\mathrm{i} m \theta_{j}\right) .
\end{aligned}
$$

in the matrix, and likewise in the fibers based on Eqs. (11) and (12).

The displacement fields in the matrix are given by the sum of those corresponding to the incident wave and the scattered waves, i.e. $\mathbf{u}(\mathbf{r})=\mathbf{u}^{\text {inc }}(\mathbf{r})+\mathbf{u}^{\text {sca }}(\mathbf{r})$ in $D_{1}$. The displacements of the incident wave are given by Eqs. (2), (5) and (6) as

$$
u_{1}^{\text {inc }}(\mathbf{r})=\mathrm{i} k_{\mathrm{L} 1} \Phi_{0} \exp \left(\mathrm{i} k_{\mathrm{L} 1} x_{1}\right), \quad u_{2}{ }^{\text {inc }}(\mathbf{r})=0,
$$

for the $\mathrm{P}$ wave and

$$
u_{1}^{\text {inc }}(\mathbf{r})=0, \quad u_{2}^{\text {inc }}(\mathbf{r})=-\mathrm{i} k_{\mathrm{T} 1} \Psi_{0} \exp \left(\mathrm{i} k_{\mathrm{T} 1} x_{1}\right),
$$

for the SV wave. On the other hand, the displacements of the scattered waves are first expressed for each fiber in each local polar coordinates, converted to Cartesian components, and finally summed up for $N$ fibers as 


$$
\begin{aligned}
u_{1}{ }^{\mathrm{sca}}(\mathbf{r}) & =\sum_{i=1}^{N} \sum_{n=-\infty}^{\infty}\left[\left\{k_{\mathrm{L} 1} A_{n}{ }^{i} H_{n}^{\prime}\left(k_{\mathrm{L} 1}\left|\mathbf{r}-\mathbf{r}_{i}\right|\right)+\frac{\mathrm{i} n B_{n}{ }^{i}}{\left|\mathbf{r}-\mathbf{r}_{i}\right|} H_{n}\left(k_{\mathrm{T} 1}\left|\mathbf{r}-\mathbf{r}_{i}\right|\right)\right\} \cos \theta_{i}\right. \\
& \left.-\left\{\frac{\operatorname{in} A_{n}{ }^{i}}{\left|\mathbf{r}-\mathbf{r}_{i}\right|} H_{n}\left(k_{\mathrm{L} 1}\left|\mathbf{r}-\mathbf{r}_{i}\right|\right)-k_{\mathrm{T} 1} B_{n}{ }^{i} H_{n}^{\prime}\left(k_{\mathrm{T} 1}\left|\mathbf{r}-\mathbf{r}_{i}\right|\right)\right\} \sin \theta_{i}\right] \exp \left(\operatorname{in} \theta_{i}\right) \\
u_{2}{ }^{\text {sca }}(\mathbf{r}) & =\sum_{i=1}^{N} \sum_{n=-\infty}^{\infty}\left[\left\{k_{\mathrm{L} 1} A_{n}{ }^{i} H_{n}^{\prime}\left(k_{\mathrm{L} 1}\left|\mathbf{r}-\mathbf{r}_{i}\right|\right)+\frac{\mathrm{i} n B_{n}{ }^{i}}{\left|\mathbf{r}-\mathbf{r}_{i}\right|} H_{n}\left(k_{\mathrm{T} 1}\left|\mathbf{r}-\mathbf{r}_{i}\right|\right)\right\} \sin \theta_{i}\right. \\
& \left.+\left\{\frac{\operatorname{in} A_{n}{ }^{i}}{\left|\mathbf{r}-\mathbf{r}_{i}\right|} H_{n}\left(k_{\mathrm{L} 1}\left|\mathbf{r}-\mathbf{r}_{i}\right|\right)-k_{\mathrm{T} 1} B_{n}{ }^{i} H_{n}^{\prime}\left(k_{\mathrm{T} 1}\left|\mathbf{r}-\mathbf{r}_{i}\right|\right)\right\} \cos \theta_{i}\right] \exp \left(\operatorname{in} \theta_{i}\right)
\end{aligned}
$$

The displacement fields in the ith fiber can be obtained as

$$
\begin{aligned}
u_{1}^{i, \text { ref }}(\mathbf{r}) & =\sum_{n=-\infty}^{\infty}\left[\left\{k_{\mathrm{L} 2} C_{n}{ }^{i} J_{n}^{\prime}\left(k_{\mathrm{L} 2}\left|\mathbf{r}-\mathbf{r}_{i}\right|\right)+\frac{\mathrm{in} D_{n}{ }^{i}}{\left|\mathbf{r}-\mathbf{r}_{i}\right|} J_{n}\left(k_{\mathrm{T} 2}\left|\mathbf{r}-\mathbf{r}_{i}\right|\right)\right\} \cos \theta_{i}\right. \\
& \left.-\left\{\frac{i n C_{n}^{i}}{\left|\mathbf{r}-\mathbf{r}_{i}\right|} J_{n}\left(k_{\mathrm{L} 2}\left|\mathbf{r}-\mathbf{r}_{i}\right|\right)-k_{\mathrm{T} 2} D_{n}{ }^{i} J_{n}^{\prime}\left(k_{\mathrm{T} 2}\left|\mathbf{r}-\mathbf{r}_{i}\right|\right)\right\} \sin \theta_{i}\right] \exp \left(\mathrm{in} \theta_{i}\right) \\
u_{2}^{i, \text { ref }}(\mathbf{r}) & =\sum_{n=-\infty}^{\infty}\left[\left\{k_{\mathrm{L} 2} C_{n}{ }^{i} J_{n}^{\prime}\left(k_{\mathrm{L} 2}\left|\mathbf{r}-\mathbf{r}_{i}\right|\right)+\frac{\mathrm{in} D_{n}{ }^{i}}{\left|\mathbf{r}-\mathbf{r}_{i}\right|} J_{n}\left(k_{\mathrm{T} 2}\left|\mathbf{r}-\mathbf{r}_{i}\right|\right)\right\} \sin \theta_{i}\right. \\
& \left.+\left\{\frac{i n C_{n}{ }^{i}}{\left|\mathbf{r}-\mathbf{r}_{i}\right|} J_{n}\left(k_{\mathrm{L} 2}\left|\mathbf{r}-\mathbf{r}_{i}\right|\right)-k_{\mathrm{T} 2} D_{n}{ }^{i} J_{n}^{\prime}\left(k_{\mathrm{T} 2}\left|\mathbf{r}-\mathbf{r}_{i}\right|\right)\right\} \cos \theta_{i}\right] \exp \left(\operatorname{in} \theta_{i}\right)
\end{aligned}
$$

The stress fields can be calculated likewise, but their explicit expressions are lengthy and omitted here.

\subsection{Numerical implementation}

In the present numerical analysis, our intension is to model an infinite number of fibers embedded in the region covering $0<x_{1}<L$ and extending infinitely in the $x_{2}$-direction in the matrix. To this purpose, the idea of repeated fundamental blocks including a finite number of fibers as utilized in [25] is also employed here. Namely, the arrangement of $N_{\mathrm{f}}$ fibers in a rectangular region $0<x_{1}<L$ and $0<x_{2}<H$, called the fundamental block, is repeated in the $x_{2}$-direction to construct the whole fiber arrangement (Fig. 2). This assumption allows us to consider that the wave field is periodic in the $x_{2}$-direction with period $H$, so that the coefficients $E_{n}{ }^{i}$ and $F_{n}{ }^{i}$ are the same for the corresponding fibers in all blocks. In this circumstance Eqs. (15a, b) can be summarized for $N_{\mathrm{f}}$ fibers in a single fundamental block $0<x_{1}<L$ and $0<x_{2}<H$, yielding

$$
\begin{aligned}
& \sum_{n=-\infty}^{\infty} E_{n}{ }^{i} J_{n}\left(k_{\mathrm{L} 1}\left|\mathbf{r}-\mathbf{r}_{i}\right|\right) \exp \left(\mathrm{in} \theta_{i}\right) \\
=\Phi^{\mathrm{inc}}(\mathbf{r}) & +\sum_{\substack{p=-\infty \\
p \neq 0}}^{\infty} \sum_{m=-\infty}^{\infty}\left(S_{m} E_{m}{ }^{i}+T_{m} F_{m}{ }^{i}\right) H_{m}\left(k_{\mathrm{L} 1}\left|\mathbf{r}-\left(\mathbf{r}_{i}+p H \mathbf{n}_{2}\right)\right|\right) \exp \left(\mathrm{i} m \theta_{i p}\right) \\
& +\sum_{p=-\infty}^{\infty} \sum_{\substack{j=1 \\
j \neq i}}^{N_{\mathrm{f}}} \sum_{m=-\infty}^{\infty}\left(S_{m} E_{m}{ }^{j}+T_{m} F_{m}{ }^{j}\right) H_{m}\left(k_{\mathrm{L} 1}\left|\mathbf{r}-\left(\mathbf{r}_{j}+p H \mathbf{n}_{2}\right)\right|\right) \exp \left(\mathrm{i} m \theta_{j p}\right),
\end{aligned}
$$




$$
\begin{aligned}
& \sum_{n=-\infty}^{\infty} F_{n}{ }^{i} J_{n}\left(k_{\mathrm{T} 1}\left|\mathbf{r}-\mathbf{r}_{i}\right|\right) \exp \left(\mathrm{in} \theta_{i}\right) \\
& =\Psi^{\mathrm{inc}}(\mathbf{r})+\sum_{\substack{p=-\infty \\
p \neq 0}}^{\infty} \sum_{m=-\infty}^{\infty}\left(U_{m} E_{m}{ }^{i}+V_{m} F_{m}{ }^{i}\right) H_{m}\left(k_{\mathrm{T} 1}\left|\mathbf{r}-\left(\mathbf{r}_{i}+p H \mathbf{n}_{2}\right)\right|\right) \exp \left(\mathrm{i} m \theta_{i p}\right) \\
& \quad+\sum_{p=-\infty}^{\infty} \sum_{\substack{j=1 \\
j \neq i}}^{N_{\mathrm{f}}} \sum_{m=-\infty}^{\infty}\left(U_{m} E_{m}{ }^{j}+V_{m} F_{m}{ }^{j}\right) H_{m}\left(k_{\mathrm{T} 1}\left|\mathbf{r}-\left(\mathbf{r}_{j}+p H \mathbf{n}_{2}\right)\right|\right) \exp \left(\mathrm{i} m \theta_{j p}\right),
\end{aligned}
$$

which hold in the matrix region of the fundamental block. In the above expressions, $\mathbf{n}_{2}$ is the unit vector in the $x_{2}$-direction, and $\theta_{i p}$ is the polar angle of a generic point $\mathbf{r}$ viewed from the center of the ith fiber in the $p$ th block, $\mathbf{r}_{i}+p H \mathbf{n}_{2}(p=0, \pm 1, \pm 2, \ldots \ldots)$.

In order to determine the coefficients $E_{n}{ }^{i}$ and $F_{n}{ }^{i}$ satisfying Eqs. (21) by numerical means, the infinite series of the eigenfunction expansions are truncated at a certain number for $n$ or $m$. Namely, these infinite sums are replaced by finite ones with $n$ (or $m)=0, \pm 1, \pm 2, \ldots, \pm n_{\max }$. Also, the summation for infinite blocks (over $p$ in Eq. (21)) is also replaced by a finite one, namely for $p=0, \pm 1, \pm 2, \ldots, \pm p_{\max }$. The truncation parameters $n_{\max }$ and $p_{\max }$ are chosen to be sufficiently large in the numerical analysis so that further increase of their respective value makes negligible effect on the solution. Then, there are $2 N_{\mathrm{f}}$ $\left(2 n_{\max }+1\right)$ unknown variables $E_{n}{ }^{i}$ and $F_{n}{ }^{i}$. Since Eqs. (21) are satisfied at an arbitrary point $\mathbf{r}$ in the region occupied by the matrix, by choosing an appropriate number of collocation points one obtains a set of linear equations for these coefficients. In principle, $\left(2 n_{\max }+1\right)$ collocation points can be chosen for each fiber to construct a set of linear equations for the unknown variables. In the present analysis, however, it has been observed that the coefficient matrix of such a system becomes ill-conditioned for certain fiber arrangements and frequencies. Therefore, in order to obtain the solutions robustly, $\left(2 n_{\max }+2\right)$ collocation points are employed at equidistant points on the boundary of each fibers, thus constructing an over-determined system of linear equations. The obtained set of equations is solved by using the generalized inverse matrix. Once the expansion coefficients are obtained numerically, it is straightforward to compute the potentials as well as the displacement fields for both matrix and fibers.

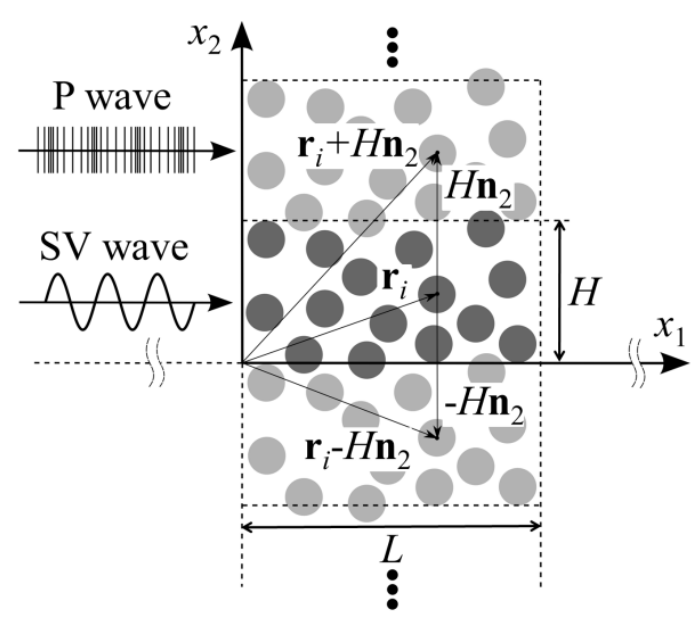

Fig. 2 Construction of a numerical model by fundamental blocks. 


\section{Scope of numerical analysis}

\subsection{Material parameters}

As specific and demonstrative examples of the two-dimensional multiple scattering of elastic waves in fibrous composites, the wave propagation characteristics in unidirectional SiC-fiber reinforced Ti-alloy composites are considered. Here, both fibers (radius 70 micrometers) and matrix are assumed isotropic in the $x_{1}-\chi_{2}$ plane and linear elastic, and the material parameters are set as shown in Table 1 . Although it is known that the third phase (coating layer) is often introduced at the matrix-fiber interfaces in such composites [34], the fibers are assumed to be directly bonded to the matrix in the present analysis. It is straightforward though to incorporate a concentric interphase zone or a spring-type interface imperfection into the analysis, which merely modifies the explicit form of the matrix $\left[M^{(n)}\right]$ in Eq. (12).

Two fundamental characteristics of elastic waves in unidirectional composites are examined in this paper, namely, (i) the effective phase velocities of the composite at relatively low frequencies, and (ii) the energy transmission spectra of the composite and the presence of the stop bands. For each of these

Table 1

Material parameters for numerical analysis.

\begin{tabular}{|c|c|c|}
\hline \multicolumn{3}{|c|}{ Matrix (Ti-alloy) } \\
\hline $\begin{array}{c}\lambda_{1}(\mathrm{GPa}) \\
103\end{array}$ & $\begin{array}{c}\mu_{1}(\mathrm{GPa}) \\
44.8\end{array}$ & $\begin{array}{c}\rho_{1}\left(\mathrm{~kg} \mathrm{~m}^{-3}\right) \\
5400\end{array}$ \\
\hline \multicolumn{3}{|l|}{ Fiber (SiC) } \\
\hline $\begin{array}{c}\lambda_{2}(\mathrm{GPa}) \\
92.1\end{array}$ & $\begin{array}{c}\mu_{2}(\mathrm{GPa}) \\
177\end{array}$ & $\begin{array}{c}\rho_{2}\left(\mathrm{~kg} \mathrm{~m}^{-3}\right) \\
3200\end{array}$ \\
\hline
\end{tabular}

Table 2

Dimensions of the fundamental block with 160 fibers, for $\phi=0.25$.

\begin{tabular}{cc}
\hline \multicolumn{3}{l}{ Square arrangement } \\
\hline$L(\mathrm{~mm})$ & $H(\mathrm{~mm})$ \\
19.9 & 0.496 \\
\hline Hexagonal arrangement & \\
\hline$L(\mathrm{~mm})$ & $H(\mathrm{~mm})$ \\
21.5 & 0.462 \\
\hline Random arrangements & \\
\hline$L(\mathrm{~mm})$ & $H(\mathrm{~mm})$ \\
5.43 & 1.81 \\
\hline
\end{tabular}

Table 3

Dimensions of the fundamental block with 10 fibers, for $\phi=0.25$.

\begin{tabular}{cc}
\hline \multicolumn{2}{c}{ Square arrangement } \\
\hline$L(\mathrm{~mm})$ & $H(\mathrm{~mm})$ \\
1.24 & 0.496 \\
\hline \multicolumn{2}{l}{ Hexagonal arrangement } \\
\hline$L(\mathrm{~mm})$ & $H(\mathrm{~mm})$ \\
1.47 & 0.462 \\
\hline Random arrangements & \\
\hline$L(\mathrm{~mm})$ & $H(\mathrm{~mm})$ \\
1.36 & 0.453 \\
\hline
\end{tabular}


examinations, different volume fractions and spatial arrangements of fibers are assumed in the fundamental block. For all computations shown in this paper, the truncation parameters were set as $n_{\max }=8$ and $p_{\max }=$ 7500 after careful trials to check the convergence of the solutions.

\subsection{Effective phase velocities}

To obtain the phase velocities in the composite at low frequencies, numerical models with different fiber arrangements are employed with a fixed number of fibers $N_{\mathrm{f}}=160$ in the fundamental block, namely, (i) square arrangement, (ii) hexagonal arrangement, and (iii) random arrangements as shown in Fig. 3. Fiber arrangements with different volume fractions $\phi$ are considered by changing the size of the fundamental block. For example, the dimensions $L$ and $H$ of the fundamental block for the fiber volume fraction $\phi=$ 0.25 are shown in Table 2. Fundamental blocks with other values of the volume fraction are constructed by

(a) Square arrangement

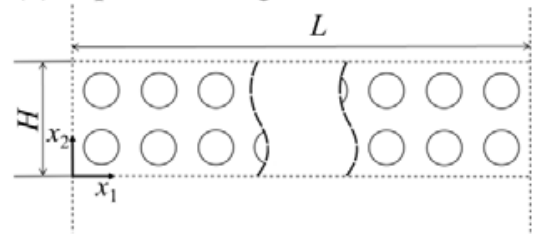

(b) Hexagonal arrangement

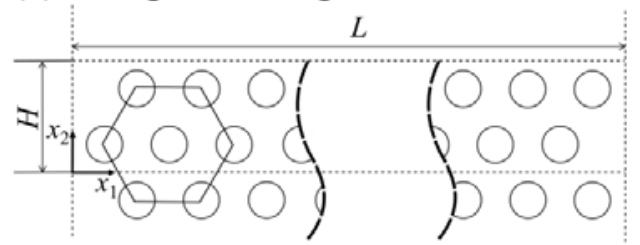

(c) Random arrangement

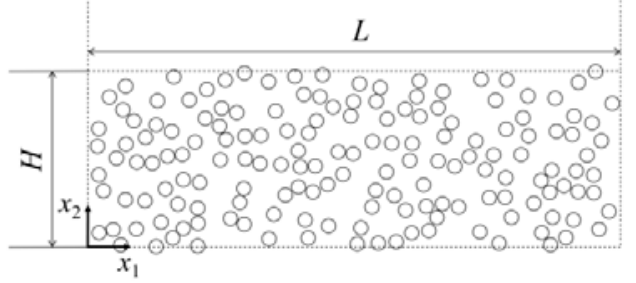

Fig. 3 (a) Square, (b) hexagonal and (c) random fiber arrangements for the analysis of the effective phase velocities.

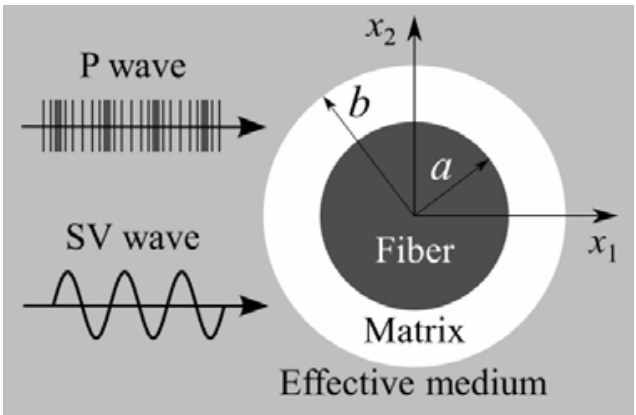

Fig. 4 Generalized self-consistent model for a unidirectional composite. 
changing these dimensions while keeping the number of the fibers and the ratio $L / H$ unchanged. For the random arrangements, a total of eight patterns are constructed for each volume fraction by a random sequential adsorption algorithm [35], with an additional condition that the neighboring fibers are always separated by

$$
\left|\mathbf{r}_{i}-\mathbf{r}_{j}\right| \geq 2.01 a, \quad(i \neq j)
$$

Furthermore, the frequency $f=\omega /(2 \pi)$ is varied between 1 and $4 \mathrm{MHz}\left(0.024<a f / c_{\mathrm{T} 1}<0.097\right.$ in a non-dimensional measure).

For the above models, the multiple scattering simulations are performed for the incidence of plane $\mathrm{P}$ and SV waves. The resulting distributions of the displacement $\left(\operatorname{Re}\left[u_{1}\right]\right.$ for the P-wave incidence and $\operatorname{Re}\left[u_{2}\right]$ for the SV-wave incidence) along the $x_{1}$-direction, at $x_{2}=H / 2$, are fitted by sinusoidal functions to extract the effective wavelengths $\lambda_{\mathrm{L}}$ and $\lambda_{\mathrm{T}}$ in the reinforced region $0<x_{1}<L$, from which the effective phase velocities $c_{\mathrm{L}}$ and $c_{\mathrm{T}}$ of the composite are obtained by the relations $c_{\mathrm{L}}=f \lambda_{\mathrm{L}}$ and $c_{\mathrm{T}}=f \lambda_{\mathrm{T}}$. The influence of the fiber arrangement, the fiber volume fraction and the frequency on the effective phase velocities of the composite is then examined. It is noted in passing that as shown in Table 2, a greater ratio $H / L$ is chosen for the random arrangements (iii) compared to the cases (i) and (ii), in order to allow certain extent of disorder of the fiber arrangement in the vertical direction while keeping $L$ long enough to evaluate the effective wavelengths.

It is of definite interest to compare the obtained effective phase velocities of the composite to the predictions of an existing theoretical model. To this purpose, the generalized self-consistent (GSC) multiple

(a) Square arrangement

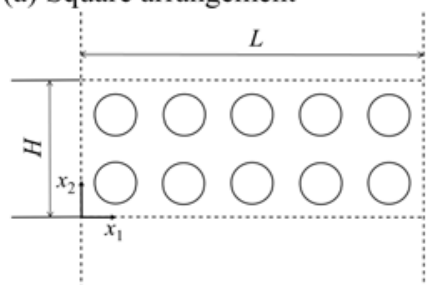

(b) Hexagonal arrangement

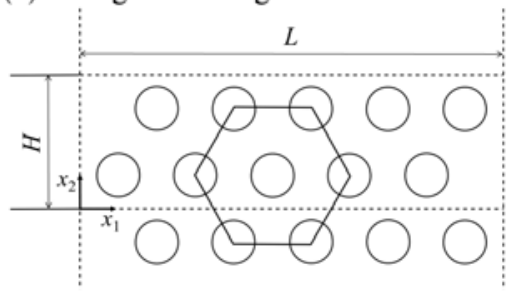

(c) Random arrangement

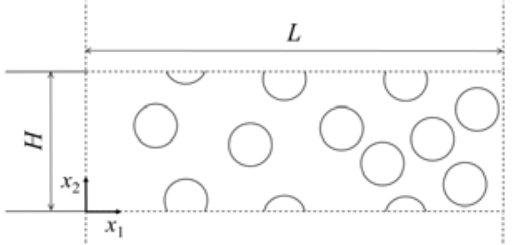

Fig. 5 (a) Square, (b) hexagonal and (c) random fiber arrangements for the analysis of the energy transmission coefficients. 
scattering model by Yang and Mal [16] is chosen among others, in which the fiber-reinforced composite is modeled as an effective medium containing a single fiber and the surrounding matrix shell of the outer radius $b$ determined by $(a / b)^{2}=\phi$, as shown in Fig. 4 . This composite fiber is subjected to a plane wave incidence, and the effective wave numbers of the composite are determined by an iterative procedure, from which the effective phase velocities are extracted.

\subsection{Energy transmission characteristics}

When the wavelength of the propagating wave in the composite is of a comparable order to the fiber radius or the fiber spacing, the wave interacts with the fibers in a complex fashion. A particular situation arises when the fibers are arranged in a regular manner. For certain bands of the frequency, the reflected waves from different fibers interfere constructively and the propagating wave exhibits an exponential decay. Using the formulation of the present type, this phenomenon has been analyzed for scalar SH waves in unidirectional composites [26, 27], and now the analysis is extended to two-dimensional elastodynamic problems involving $\mathrm{P}$ and SV waves.

To this purpose, the fundamental blocks as shown in Fig. 5 are employed, with (a) square and (b) hexagonal arrangements with 10 fibers in the fundamental block. In order to see the effect of perturbed fiber arrangements, the simulations are also performed for eight patterns of the same number of fibers which are randomly arranged in the fundamental block, one of which is shown in Fig. 5 (c). It is noted, however, that these arrangements do not correspond to those of an ideally random microstructure, due to the small number of fibers assumed in the fundamental block: furthermore, the periodicity of the length $H$ is always persistent in the $x_{2}$-direction. The dimensions of the fundamental block for these arrangements are shown in Table 3 in the case of $\phi=0.25$. While a longer fundamental block and a greater number of fibers are employed for the effective velocity evaluation, the smaller length of $L$ is employed here to analyze the energy transmission characteristics.

From the displacement and stress fields computed by the multiple scattering simulations, the energy flux density in the $x_{1}$-direction, averaged for the $x_{2}$-direction, is obtained by

$$
E\left(x_{1}\right)=\frac{1}{H} \int_{0}^{H} P_{1}\left(x_{1}, x_{2}\right) d x_{2},
$$

where $P_{1}$ is the component of the Umov-Poynting vector in the $x_{1}$-direction given by

$$
P_{1}\left(x_{1}, x_{2}\right)=-\frac{i \omega}{4}\left(\sigma_{11} u_{1}^{*}-\sigma_{11}^{*} u_{1}+\sigma_{12} u_{2}^{*}-\sigma_{12}^{*} u_{2}\right),
$$

using the displacement and stress components in the complex-valued formulation. Then, the energy transmission coefficient can be calculated as the ratio of the averaged energy flux density, presently computed at $x_{1}=2 L$, to the energy flux density of the incident wave. The frequency dependence of the energy transmission coefficients are investigated for the range $0<f<10[\mathrm{MHz}]\left(0<a f / c_{\mathrm{T} 1}<0.24\right)$.

\section{Results and discussion}

\subsection{Wave fields in the composite}

Prior to the discussion of the effective phase velocities and the energy transmission characteristics, the numerical results are first visualized to examine the wave fields in the composite. For two representative 
frequencies of 1 and $8 \mathrm{MHz}$ ( $a f / c_{\mathrm{T} 1}=0.024$ and 0.192 , respectively), the distributions of the displacement components, $\operatorname{Re}\left[u_{1}(\mathbf{r})\right]$ and $\operatorname{Re}\left[u_{2}(\mathbf{r})\right]$, are shown in Figs. 6 to 8, for the square, hexagonal and one pattern of random arrangements, respectively, of the 160-fiber fundamental blocks as employed for the analysis of the effective phase velocities. The distributions are shown for the whole fundamental block $\left(0<x_{1}<L, 0<x_{2}\right.$ $<H$ ) for the $1-\mathrm{MHz}$ case, and for its quarter part $\left(0<x_{1}<L / 4,0<x_{2}<H\right)$ for the 8-MHz case due to the shorter wavelengths.

In each of Figs. 6-8, the wave fields shown in (a) and (b) for the 1-MHz P and SV wave incidence basically show a plane-wave nature, essentially exhibiting only the $u_{1}\left(u_{2}\right)$ component for the P (SV) wave incidence. At this frequency, the effect of the presence of fibers is to elongate the wavelengths in the composite as compared to those in pure matrix, but their arrangements do not appear to affect the local wave fields significantly.

At the higher frequency of $8 \mathrm{MHz}$, the displacement distributions show more complex behavior. Namely, for the P (SV) wave incidence, the $u_{1}\left(u_{2}\right)$ component shows some perturbation from the plane wave, and the distributions of the $u_{2}\left(u_{1}\right)$ component are clearly visible. Furthermore, the local wave fields are different for different fiber arrangements, thus showing the influence of the composite microstructure. In particular, the wave fields for the hexagonal arrangement due to the SV wave incidence, Fig. 7 (d), exhibit a decaying feature against the distance along the wave incidence direction, as a consequence of the stop-band formation which will be discussed further in the subsequent section. The corresponding case of the random arrangement, Fig. 8 (c) and (d), also shows complicated features of wave fields, revealing a significant contribution of non-propagating (standing) waves due to the local interactions. In Fig. 8 (c) and (d), the somewhat structured patterns are probably due to the finite length $H$ in the vertical direction.

(a) $1 \mathrm{MHz} \mathrm{P}$ wave incidence

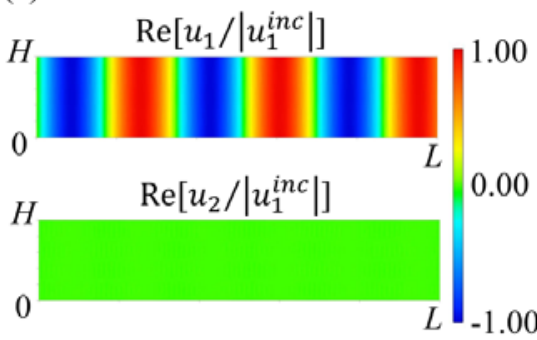

(c) $8 \mathrm{MHz}$ P wave incidence

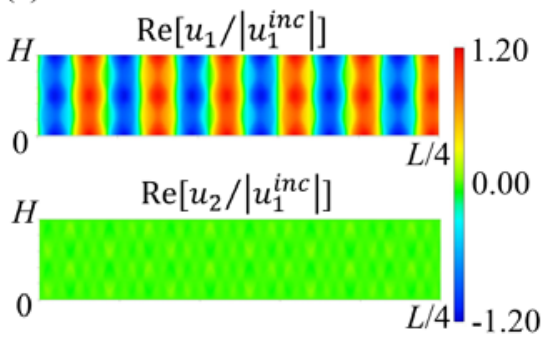

(b) $1 \mathrm{MHz} \mathrm{SV}$ wave incidence

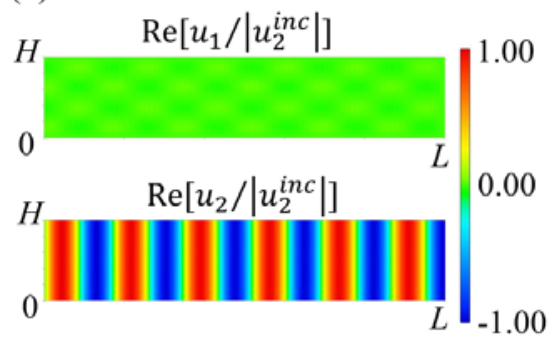

(d) $8 \mathrm{MHz}$ SV wave incidence

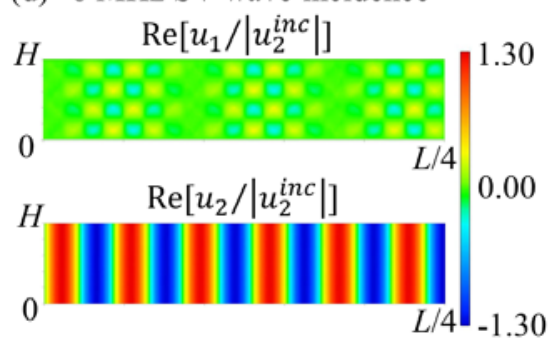

Fig. 6 Distributions of the wave displacements for the square fiber arrangement, (a), (b) for $1 \mathrm{MHz}$ $\left(a f / c_{\mathrm{T} 1}=0.024\right)$ and (c), (d) for $8 \mathrm{MHz}\left(a f / c_{\mathrm{T} 1}=0.192\right)$. 
The present multiple scattering simulations have thus demonstrated detailed interactions of elastic waves with the reinforcing fibers, accompanying the mode conversion between $\mathrm{P}$ and SV waves and

(a) $1 \mathrm{MHz} \mathrm{P}$ wave incidence

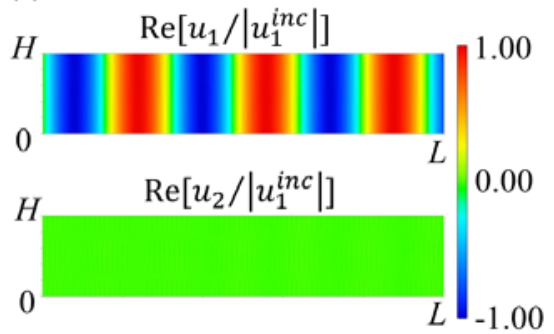

(c) $8 \mathrm{MHz} \mathrm{P}$ wave incidence

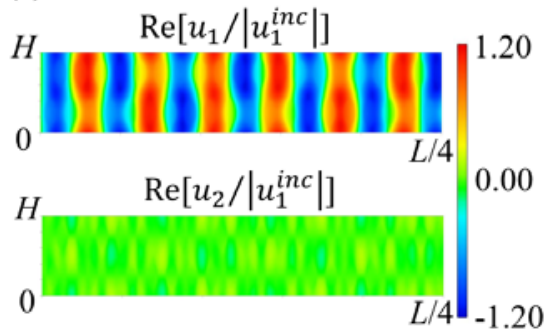

(b) $1 \mathrm{MHz} \mathrm{SV}$ wave incidence

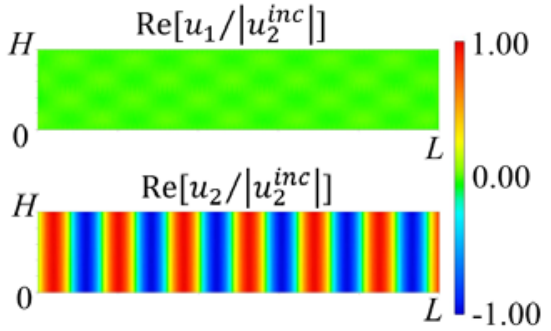

(d) $8 \mathrm{MHz} \mathrm{SV}$ wave incidence

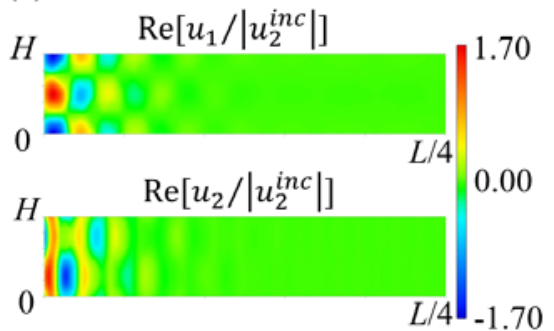

Fig. 7 Distributions of the wave displacements for the hexagonal fiber arrangement, (a), (b) for $1 \mathrm{MHz}$ $\left(a f / c_{\mathrm{T} 1}=0.024\right)$ and (c), (d) for $8 \mathrm{MHz}\left(a f / c_{\mathrm{T} 1}=0.192\right)$.

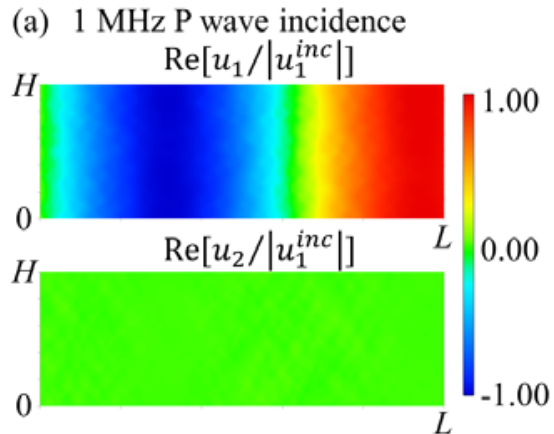

(c) $8 \mathrm{MHz}$ P wave incidence

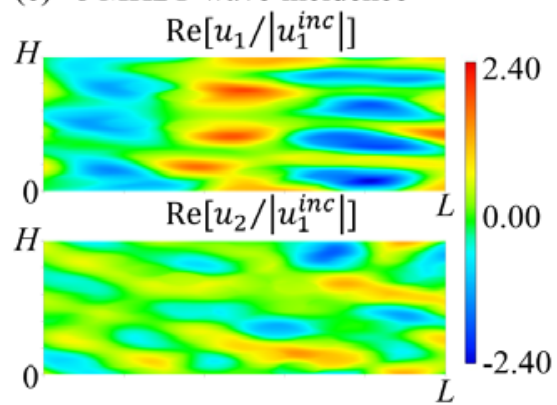

(b) $1 \mathrm{MHz} \mathrm{SV}$ wave incidence

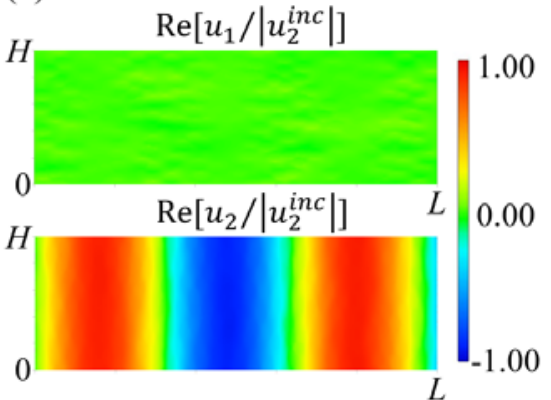

(d) $8 \mathrm{MHz} \mathrm{SV}$ wave incidence

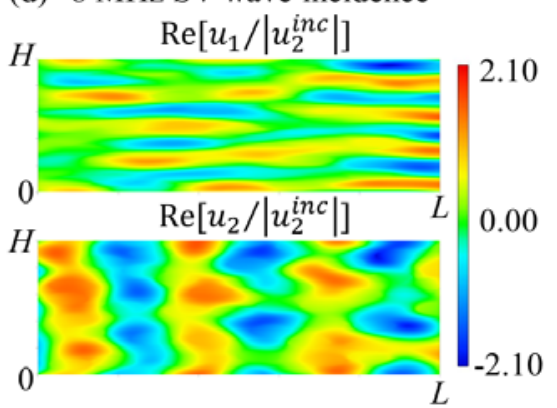

Fig. 8 Distributions of the wave displacements for the random fiber arrangement, (a), (b) for $1 \mathrm{MHz}$ $\left(a f / c_{\mathrm{T} 1}=0.024\right)$ and (c), (d) for $8 \mathrm{MHz}\left(a f / c_{\mathrm{T} 1}=0.192\right)$. 
different local wave fields in the composite depending on the fiber arrangement.

\subsection{Effective phase velocities}

The effective phase velocities $c_{\mathrm{L}}$ and $c_{\mathrm{T}}$ of the $\mathrm{P}$ and SV waves in the composite are plotted in Fig. 9 against the normalized frequency for a fixed fiber volume fraction $(\phi=0.25)$ but for different fiber arrangements, together with the predictions of the GSC model [16]. Both longitudinal and transverse phase velocities of the composite are normalized by the transverse wave speed of the matrix $c_{\mathrm{T} 1}$. For the random arrangements, the averages of the effective velocities from the simulations of eight patterns are shown. Rigorously speaking, multiple scattering theories yield the effective velocities for the averaged fields, not the averages of the phase velocities for the fields of particular fiber arrangements. For the relatively low-frequency range discussed here, however, the difference between these two quantities is considered to be insignificant. In the frequency range analyzed here, the dispersive features are not significant, and the effective phase velocities of the composite take more or less frequency-independent values. This is considered to be a consequence of relatively long wavelength as compared to the fiber radius or spacing for the frequency range plotted here. The effective phase velocities are also plotted against the fiber volume fraction in Fig. 10, for a fixed frequency of $3 \mathrm{MHz}\left(a f / c_{\mathrm{T} 1}=0.072\right)$. It is clearly seen here that as the fiber
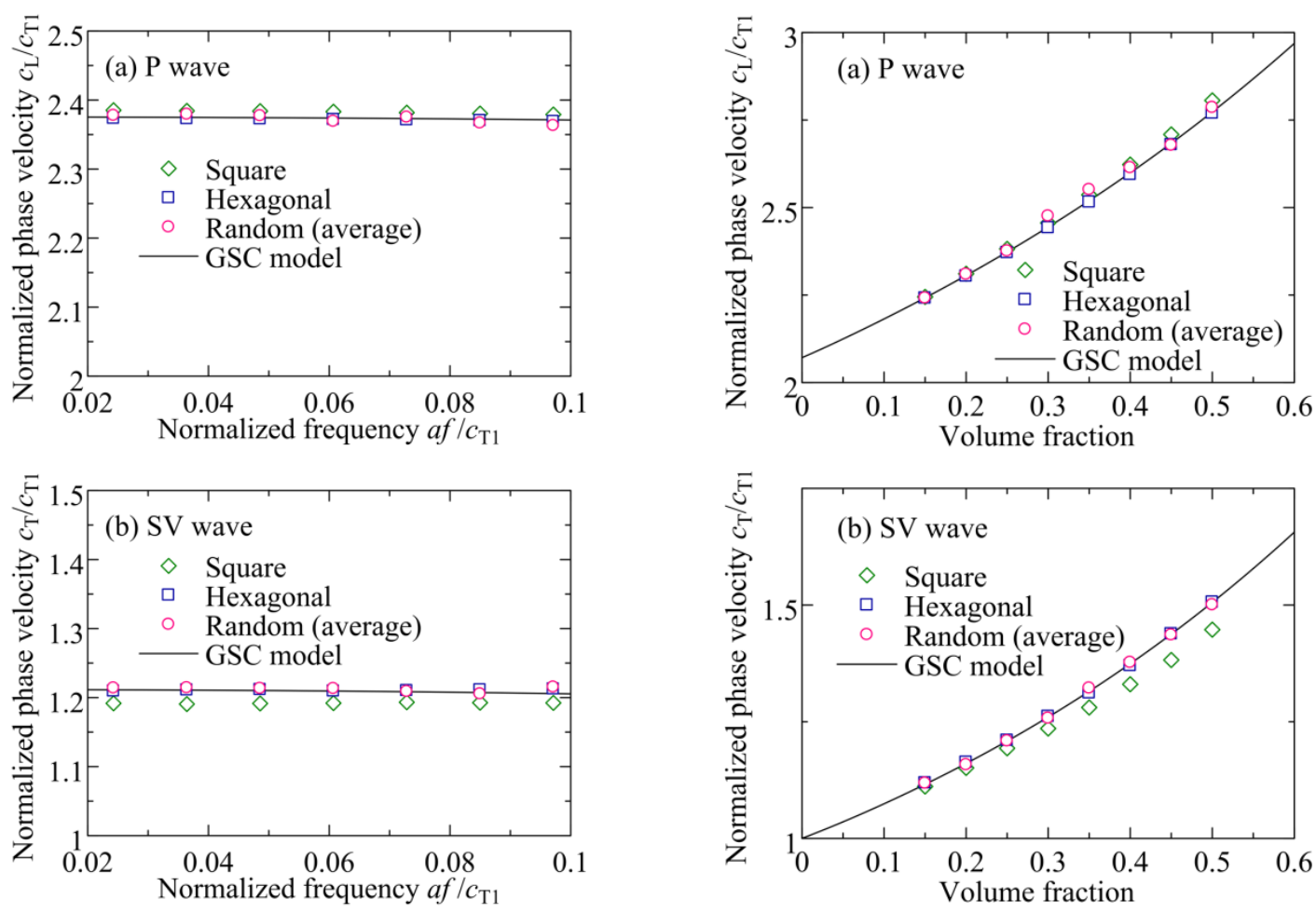

Fig. 9 Variation of the normalized (a) longitudinal and (b) shear wave velocities with the normalized frequency, for $\phi=0.25$.
Fig. 10 Variation of the normalized (a) longitudinal and (b) shear wave velocities with the fiber volume fraction, for $3 \mathrm{MHz}$ $\left(a f / c_{\mathrm{T} 1}=0.072\right)$. 
volume fraction increases both the P and SV wave velocities monotonically increase.

In both Figs. 9 and 10, the theoretical predictions by the GSC model give a good agreement with the present multiple scattering simulations, especially with the results for the hexagonal and random fiber arrangements. The agreement of the GSC model predictions with the random case could be expected at the outset. Furthermore, the hexagonal arrangement is expected to show isotropic features as long as macroscopic elastic properties are concerned, which is considered to explain the close agreement with the GSC model. For the square arrangement, as $\phi$ increases the $\mathrm{P}$ wave velocity becomes slightly higher, and the SV wave velocity lower, than the corresponding velocities for other fiber arrangements and the GSC model, indicating an anisotropic response.

\subsection{Energy transmission characteristics}

The frequency dependence of the computed energy transmission coefficients of the P and SV waves are depicted in Figs. 11 and 12, for the square and hexagonal fiber arrangements, respectively, with different fiber volume fractions. For the square fiber arrangement and the same material parameters, the elastic wave transmission characteristics were analyzed by Nakashima et al. [31] by the finite element method. Their finite element solutions and the present simulations are in good agreement regarding the energy transmission spectra, partly verifying the accuracy of both techniques.
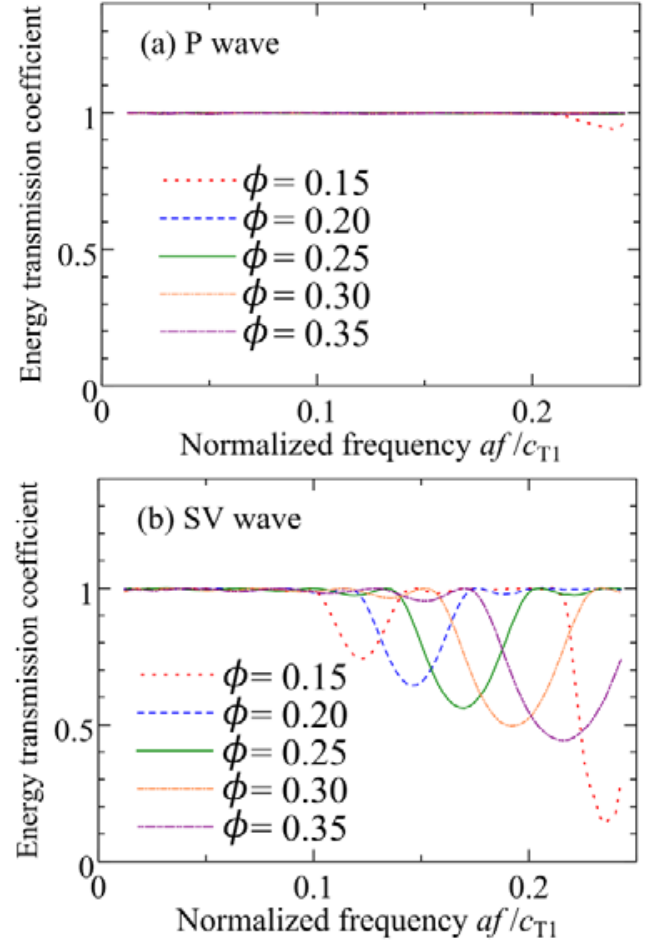

Fig. 11 Variation of the energy transmission coefficients of the (a) longitudinal and (b) shear waves with the normalized frequency for the square fiber arrangement, for different fiber volume fractions.
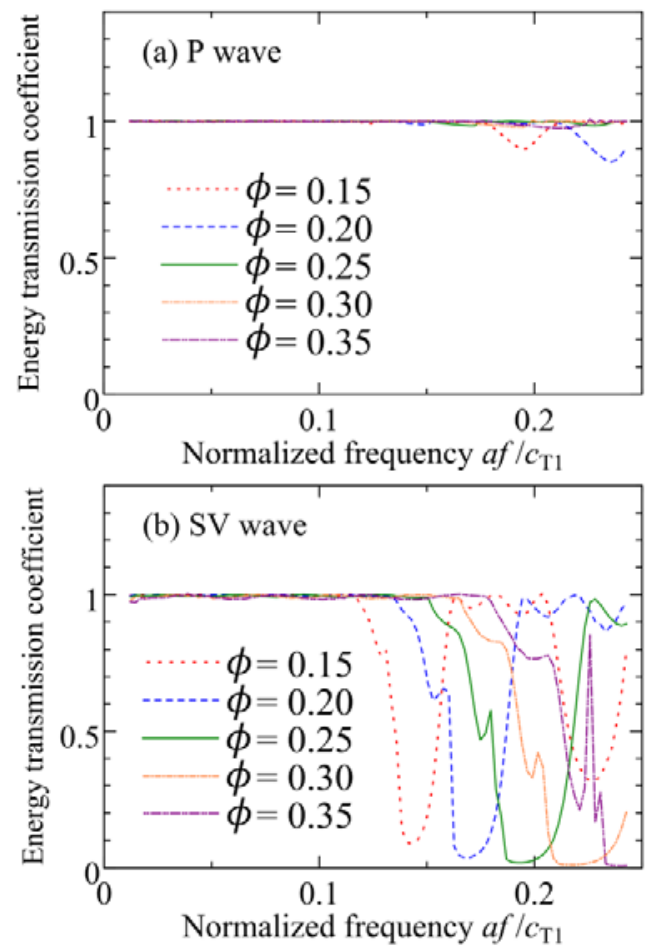

Fig. 12 Variation of the energy transmission coefficients of the (a) longitudinal and (b) shear waves with the normalized frequency for the hexagonal fiber arrangement, for different fiber volume fractions. 
Figures 11 and 12 show that while the $\mathrm{P}$ wave transmission coefficient is nearly unity in the frequency range $a f / c_{\mathrm{T} 1}<0.2$ (below $8 \mathrm{MHz}$ ), the $\mathrm{SV}$ wave shows richer transmission spectra due to its shorter wavelength than that of the P wave. The energy transmission spectra of the SV wave are found to have clear dips, at different frequencies for different fiber volume fractions. The occurrence and the locations of these dips can be explained in the following manner.

For a composite with regular fiber spacing, the scattered waves by different fibers can interfere constructively when the wavelength $\lambda$ in the composite meets a certain condition with the fiber spacing. In this situation, the energy transmission coefficient becomes null in a certain frequency band (stop band) for a composite with infinitely repeated periodic microstructure. For a composite with finite number of fibers as analyzed here, the transmission coefficient takes a non-zero but relatively low value at the corresponding

(a) Square
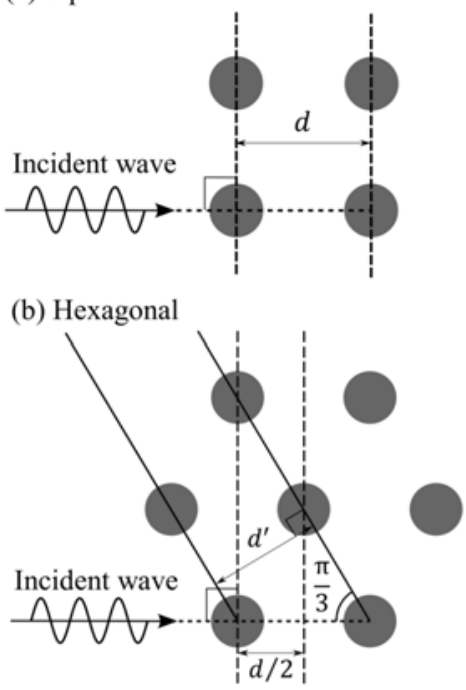

Fig. 13 Schematic representation of fiber arrays for the stop-band formation.

Table 4

The estimated cut-off frequencies for the range $0<a f_{\mathrm{C}} / c_{\mathrm{T} 1}<0.25$.

Square arrangement

$\begin{array}{cccc}\phi & d / a & c_{\mathrm{T}} / c_{\mathrm{T} 1} & a f_{\mathrm{C}} / c_{\mathrm{T} 1}\end{array}$

$\begin{array}{llll}0.15 & 4.58 & 1.11 & 0.121\end{array}$

0.243

$\begin{array}{llll}0.2 & 3.96 & 1.15 & 0.145\end{array}$

$\begin{array}{llll}0.25 & 3.54 & 1.19 & 0.168\end{array}$

$\begin{array}{llll}0.3 & 3.24 & 1.24 & 0.191\end{array}$

\begin{tabular}{cccc}
0.35 & 3.00 & 1.28 & 0.214 \\
\hline \multicolumn{2}{l}{ Hexagonal arrangement } & & \\
\hline$\phi$ & $d^{\prime} / a$ & $c_{\mathrm{T}} / c_{\mathrm{T} 1}$ & $a f_{\mathrm{C}} / c_{\mathrm{T} 1}$ \\
& $(d / a)$ & & \\
0.15 & 4.26 & 1.12 & 0.152 \\
& $(4.92)$ & & 0.228 \\
0.2 & 3.69 & 1.16 & 0.182 \\
0.25 & 3.30 & 1.21 & 0.212 \\
0.3 & 3.01 & 1.26 & 0.242 \\
\hline
\end{tabular}


frequency. The terminology of stop band is also used in this paper to refer to this feature.

Geometrically, the matching condition for the square arrangement can be written as

$$
2 d=n \lambda,
$$

where $n$ is an integer and $d$ is the distance between the neighboring fiber centers as illustrated in Fig. 13 (a). The cut-off frequencies $f_{\mathrm{C}}$ can be roughly estimated as

$$
f_{\mathrm{C}}=\frac{c_{\mathrm{T}}}{\lambda}=\frac{n c_{\mathrm{T}}}{2 d},
$$

using Eq. (25) and the effective shear wave velocity $c_{\mathrm{T}}$ of the composite as determined by the present simulations. The results are summarized in Table 4. The cut-off frequencies for the hexagonal arrangement can be estimated, as also shown in Table 4, by the following relations;

$$
\sqrt{3} d^{\prime}=n \lambda \Rightarrow f_{\mathrm{C}}=\frac{n c_{\mathrm{T}}}{\sqrt{3} d^{\prime}},
$$

where $d^{\prime}$ ' is the distance between the planes made by the fibers as shown in Fig. 13 (b), and

$$
d=n \lambda \Rightarrow f_{\mathrm{C}}=\frac{n c_{\mathrm{T}}}{d} .
$$

The frequencies estimated by Eqs. (26) and (27) are in good agreement with the locations of the dips shown
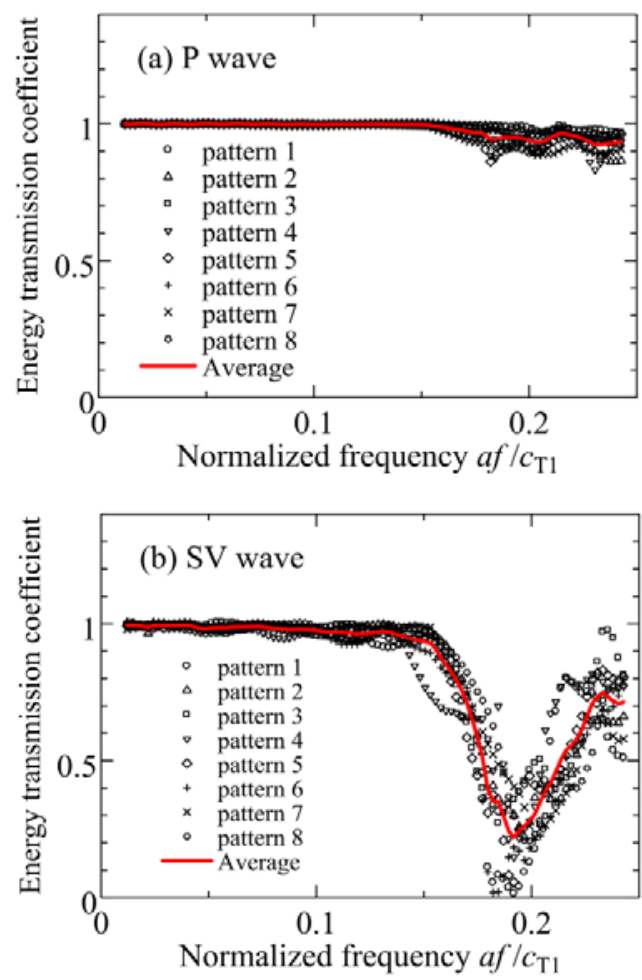

Fig. 14 Variation of the energy transmission coefficients of the (a) longitudinal and (b) shear waves with the normalized frequency for the random fiber arrangements, for $\phi=$ 0.25 .
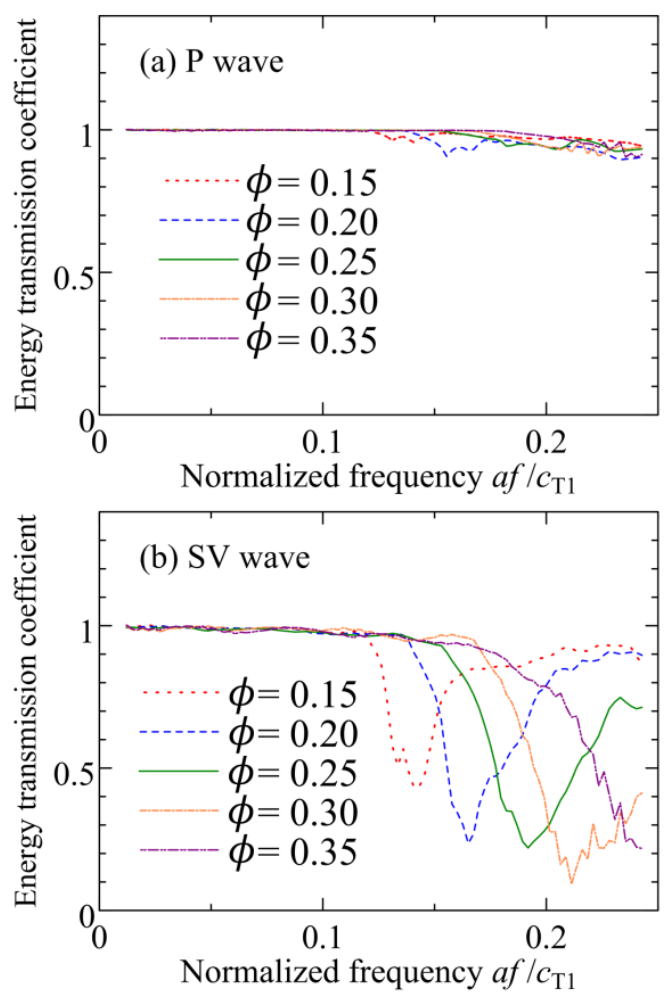

Fig. 15 Variation of the energy transmission coefficients of the (a) longitudinal and (b) shear waves with the normalized frequency for the random fiber arrangements (averaged), for different fiber volume fractions. 
in Figs. 11 and 12.

The energy transmission coefficients are also examined for random arrangements of 10 fibers in the fundamental block. In Fig. 14, eight transmission spectra corresponding to different fiber arrangements are depicted for the case $\phi=0.25$, where the averaged transmission spectrum is also shown. The averaged transmission spectra are obtained for other fiber volume fractions, and illustrated in Fig. 15. In Fig. 15 (b), the SV wave transmission spectra show dips at the frequencies close to those for the hexagonal arrangements, in spite of disordered fiber arrangements. This may be partly attributed to the relatively small number of fibers in the fundamental block and the $H$-periodicity in the $x_{2}$-direction as mentioned above. The present simulations thus indicate that for a relatively small number of fibers in the fundamental block, the stop-band features are persistent even when the fiber arrangement is disordered. Nakashima et al. [31] obtained similar finding by the finite element analysis for stacked monotapes with random misalignment. For a larger size of the fundamental block with a larger number of fibers, however, the stop-band features are expected to gradually diminish as the fiber arrangement is perturbed to randomness, as demonstrated by Biwa et al. [27] for SH waves.

\section{Conclusions}

A numerical procedure has been presented in this paper for the two-dimensional, time-harmonic elastodynamic multiple scattering problems for unidirectional fiber-reinforced composites. The P and SV wave propagation characteristics in unidirectional fiber-reinforced composites have been illustrated for different fiber arrangements and fiber volume fractions. The simulated results have been shown to clarify the detailed features of the local wave fields in the composite. The effective phase velocities of the composite have been identified and compared favorably with the predictions of a micromechanical model for random composites. The energy transmission spectra of the $\mathrm{P}$ and SV waves have been also demonstrated, which exhibit the stop-band formation for regular fiber arrangements. Stop-band features have been observed to persist in the present simulation models with limited disorder of the fiber arrangement. The numerical procedure shown here is expected to be applicable to many types of multiple scattering problems of fiber-reinforced composites and other fibrous media.

\section{Acknowledgement}

G. Haïat acknowledges the financial support from the Japan Society for the Promotion of Science (JSPS) through a JSPS Postdoctoral Fellowship which enabled him to stay at Kyoto University in 2010 and to take part in this investigation.

\section{Appendix}

In the case where a cylindrical scatterer (radius $a$ ) is perfecty bonded to the matrix so that the displacements and the tractions are continuous across the boundary, the eigenfunction expansion coefficients $A_{n}{ }^{i}, B_{n}{ }^{i}$ for the scattered potentials, $C_{n}{ }^{i}, D_{n}{ }^{i}$ for the refracted potentials, and $E_{n}{ }^{i}$ and $F_{n}{ }^{i}$ for the exciting potentials are related by 


$$
\left[\begin{array}{llll}
a_{11}^{(n)} & a_{12}^{(n)} & a_{13}^{(n)} & a_{14}^{(n)} \\
a_{21}^{(n)} & a_{22}^{(n)} & a_{23}^{(n)} & a_{24}^{(n)} \\
a_{31}^{(n)} & a_{32}^{(n)} & a_{33}^{(n)} & a_{34}^{(n)} \\
a_{41}^{(n)} & a_{42}^{(n)} & a_{43}^{(n)} & a_{44}^{(n)}
\end{array}\right]\left(\begin{array}{c}
A_{n}{ }^{i} \\
B_{n}{ }^{i} \\
C_{n}{ }^{i} \\
D_{n}{ }^{i}
\end{array}\right)=\left[\begin{array}{ll}
b_{11}^{(n)} & b_{12}^{(n)} \\
b_{21}^{(n)} & b_{22}^{(n)} \\
b_{31}^{(n)} & b_{32}^{(n)} \\
b_{41}^{(n)} & b_{42}^{(n)}
\end{array}\right]\left(\begin{array}{l}
E_{n}{ }^{i} \\
F_{n}{ }^{i}
\end{array}\right) .
$$

The elements of the above matrices are given by

$$
\begin{aligned}
& a_{11}^{(n)}=-\left\{-2 q_{\mathrm{L} 1} H_{n}^{\prime}\left(q_{\mathrm{L} 1}\right)+\left(2 n^{2}-q_{\mathrm{T} 1}{ }^{2}\right) H_{n}\left(q_{\mathrm{L} 1}\right)\right\}, \\
& a_{12}^{(n)}=-\left\{2 \mathrm{in} q_{\mathrm{T} 1} H_{n}^{\prime}\left(q_{\mathrm{T} 1}\right)-2 \mathrm{in} H_{n}\left(q_{\mathrm{T} 1}\right)\right\}, \\
& a_{13}^{(n)}=\bar{\mu}\left\{-2 q_{\mathrm{L} 2} J_{n}^{\prime}\left(q_{\mathrm{L} 2}\right)+\left(2 n^{2}-q_{\mathrm{T} 2}{ }^{2}\right) J_{n}\left(q_{\mathrm{L} 2}\right)\right\} \text {, } \\
& a_{14}^{(n)}=\bar{\mu}\left\{2 \mathrm{in} q_{\mathrm{T} 2} J_{n}^{\prime}\left(q_{\mathrm{T} 2}\right)-2 \mathrm{in} J_{n}\left(q_{T 2}\right)\right\}, \\
& a_{21}^{(n)}=-\left\{2 \mathrm{in} q_{\mathrm{L} 1} H_{n}^{\prime}\left(q_{\mathrm{L} 1}\right)-2 \mathrm{in} H_{n}\left(q_{\mathrm{L} 1}\right)\right\} \text {, } \\
& a_{22}^{(n)}=-\left\{2 q_{\mathrm{T} 1} H_{n}^{\prime}\left(q_{\mathrm{T} 1}\right)+\left(q_{\mathrm{T} 1}{ }^{2}-2 n^{2}\right) H_{n}\left(q_{\mathrm{T} 1}\right)\right\} \text {, } \\
& a_{23}^{(n)}=\bar{\mu}\left\{2 \mathrm{in} q_{\mathrm{L} 2} J_{n}^{\prime}\left(q_{\mathrm{L} 2}\right)-2 \mathrm{in} J_{n}\left(q_{\mathrm{L} 2}\right)\right\} \text {, } \\
& a_{24}^{(n)}=\bar{\mu}\left\{2 q_{\mathrm{T} 2} J_{n}^{\prime}\left(q_{\mathrm{T} 2}\right)+\left(q_{\mathrm{T} 2}{ }^{2}-2 n^{2}\right) J_{n}\left(q_{\mathrm{T} 2}\right)\right\} \text {, } \\
& a_{31}^{(n)}=-q_{L 1} H_{n}^{\prime}\left(q_{\mathrm{L} 1}\right) \text {, } \\
& a_{32}^{(n)}=-\mathrm{in} H_{n}\left(q_{\mathrm{T} 1}\right) \text {, } \\
& a_{33}^{(n)}=q_{\mathrm{L} 2} J_{n}^{\prime}\left(q_{\mathrm{L} 2}\right) \text {, } \\
& a_{34}^{(n)}=\operatorname{in} J_{n}\left(q_{\mathrm{T} 2}\right) \text {, } \\
& a_{41}^{(n)}=-\mathrm{in} H_{n}\left(q_{\mathrm{L} 1}\right), \\
& a_{42}^{(n)}=q_{\mathrm{T} 1} H_{n}^{\prime}\left(q_{\mathrm{T} 1}\right) \text {, } \\
& a_{43}^{(n)}=\operatorname{inJ} J_{n}\left(q_{\mathrm{L} 2}\right) \text {, } \\
& a_{44}^{(n)}=-q_{\mathrm{T} 2} J_{n}^{\prime}\left(q_{\mathrm{T} 2}\right), \\
& b_{11}^{(n)}=-2 q_{\mathrm{L} 1} J_{n}^{\prime}\left(q_{\mathrm{L} 1}\right)+\left(2 n^{2}-q_{\mathrm{T} 1}{ }^{2}\right) J_{n}\left(q_{\mathrm{L} 1}\right) \text {, } \\
& b_{12}^{(n)}=2 \mathrm{i} n q_{\mathrm{T} 1} J_{n}^{\prime}\left(q_{\mathrm{T} 1}\right)-2 \mathrm{i} n J_{n}\left(q_{\mathrm{T} 1}\right) \text {, } \\
& b_{21}^{(n)}=2 \mathrm{in} n q_{\mathrm{L} 1} J_{n}^{\prime}\left(q_{\mathrm{L} 1}\right)-2 \mathrm{i} n J_{n}\left(q_{\mathrm{L} 1}\right) \text {, } \\
& b_{22}^{(n)}=2 q_{\mathrm{T} 1} J_{n}^{\prime}\left(q_{\mathrm{T} 1}\right)+\left(q_{\mathrm{T} 1}{ }^{2}-2 n^{2}\right) J_{n}\left(q_{\mathrm{T} 1}\right) \text {, } \\
& b_{31}^{(n)}=q_{\mathrm{L} 1} J_{n}^{\prime}\left(q_{\mathrm{L} 1}\right) \text {, } \\
& b_{32}^{(n)}=\operatorname{in} J_{n}\left(q_{\mathrm{T} 1}\right) \text {, } \\
& b_{41}^{(n)}=\operatorname{in} J_{n}\left(q_{\mathrm{L} 1}\right) \text {, } \\
& b_{42}^{(n)}=-q_{\mathrm{T} 1} J_{n}^{\prime}\left(q_{\mathrm{T} 1}\right) \text {, }
\end{aligned}
$$

where $J_{n}$ and $H_{n}$ are the $n$ th-order Bessel and Hankel functions of the first kind, respectively, and

$$
\begin{aligned}
& q_{\mathrm{L} 1}=k_{\mathrm{L} 1} a, \quad q_{\mathrm{T} 1}=k_{\mathrm{T} 1} a, \quad q_{\mathrm{L} 2}=k_{\mathrm{L} 2} a, \quad q_{\mathrm{T} 2}=k_{\mathrm{T} 2} a, \\
& \bar{\mu}=\mu_{2} / \mu_{1}, \\
& J_{n}^{\prime}(\mathrm{z})=\frac{\mathrm{d}}{\mathrm{d} z} J_{n}(\mathrm{z}), \quad H_{n}^{\prime}(\mathrm{z})=\frac{\mathrm{d}}{\mathrm{d} z} H_{n}(\mathrm{z}) .
\end{aligned}
$$

Therefore, the matrix $\left[M^{(n)}\right]$ in eq. (12) is obtained by the matrices $\left[a^{(n)}\right]$ and $\left[b^{(n)}\right]$, i.e. $\left[M^{(n)}\right]=\left[a^{(n)}\right]^{-1}\left[b^{(n)}\right]$. 


\section{References}

[1] K. Sobczyk, Stochastic Wave Propagation, Elsevier, Amsterdam, 1985.

[2] R. A. Kline, Nondestructive Characterization of Composite Media, Technomic Pub., Pennsylvania, 1992.

[3] Y. C. Chu, S. I. Rokhlin, Fiber-matrix interphase characterization in composites using ultrasonic velocity data, J. Appl. Phys. 76 (1994), 4121-4129.

[4] S. K. Bose, A. K. Mal, Elastic waves in a fiber-reinforced composite, J. Mech. Phys. Solids 22 (1974), 217-229.

[5] V. K. Varadan, Y. Ma, V. V. Varadan, Multiple scattering of compressional and shear waves by fiber-reinforced composite materials, J. Acoust. Soc. Am. 80 (1986), 333-339.

[6] W. Liu, R. D. Kriz, Multiple wave scattering in fiber-reinforced composites: micromechanical viewpoint, Wave Motion 27 (1998), 223-244.

[7] P. A. Martin, Multiple Scattering: Interaction of Time-Harmonic Waves with N Obstacles, Cambridge University Press, Cambridge, 2006.

[8] J.-M. Conoir, A. N. Norris, Effective wavenumbers and reflection coefficients for an elastic medium containing random configurations of cylindrical scatterers, Wave Motion 47 (2010), 183-197.

[9] R. B. Nelson, P. Navi, Harmonic wave propagation in composite materials, J. Acoust. Soc. Am. 57 (1975), 773-781.

[10] T. Naciri, P. Navi, A. Ehrlacher, Harmonic wave propagation in viscoelastic heterogeneous materials: Part I. Dispersion and damping relations, Mech. Mater. 18 (1994), 313-333.

[11] T. Naciri, P. Navi, A. Ehrlacher, Harmonic wave propagation in viscoelastic heterogeneous materials: Part II. Effective complex moduli, Mech. Mater. 18 (1994), 335-350.

[12] M. S. Kushwaha, P. Halevi, G. Martínez, L. Dobrzynskí, B. Djafari-Rouhani, Theory of acoustic band structure of periodic elastic composites, Phys. Rev. B 49 (1994), 2313-2322.

[13] D. R. S. Talbot, J. R. Willis, Variational estimates for dispersion and attenuation of waves in random composites-III. Fibre-reinforced materials, Int. J. Solids Struct. 19 (1983), 793-811.

[14] H. Murakami, G. A. Hegemier, A mixture model for unidirectionally fiber-reinforced composites, Trans. ASME J. Appl. Mech. 53 (1986), 765-773.

[15] A. I. Beltzer, The effective dynamic response of random composites and polycrystals: a survey of the causal approach, Wave Motion 11 (1989), 211-229.

[16] R.-B. Yang, A. K. Mal, Multiple scattering of elastic waves in a fiber-reinforced composite, J. Mech. Phys. Solids 42 (1994), 1945-1968.

[17] J. T. Verbis, S. E. Kattis, S. V. Tsinopoulos, D. Polyzos, Wave dispersion and attenuation in fiber composites, Comp. Mech. 27 (2001), 244-252.

[18] S. Biwa, Y. Watanabe, N. Ohno, Analysis of wave attenuation in unidirectional viscoelastic composites by a differential scheme, Compos. Sci. Tech. 63 (2003), 237-247.

[19] J.-Y. Kim, On the generalized self-consistent model for elastic wave propagation in composite materials, Int. J. Solids Struct. 41 (2004), 4349-4360.

[20] D. M. Elzey, H. N. G. Wadley, Modeling the densification of metal matrix composite monotape, Acta Metall. Mater. 41 (1993), 2297-2316. 
[21] Z. X. Guo, B. Derby, Fibre uniformity and cavitation during the consolidation of metal-matrix composite via fibre-mat and matrix foil diffusion bonding, Acta Metall. Mater. 41 (1993), 3257-3266.

[22] L.-W. Cai, J. H. Williams Jr., Large-scale multiple scattering problems, Ultrasonics 37 (1999), 453-462.

[23] L.-W. Cai, J. H. Williams Jr., Full-scale simulations of elastic wave scattering in fiber-reinforced composites, Ultrasonics 37 (1999), 463-482.

[24] L.-W. Cai, J. H. Williams Jr, NDE via stop band formation in fiber reinforced composites having square fiber arrangements, Ultrasonics 37 (1999), 483-492.

[25] S. Biwa, S. Yamamoto, F. Kobayashi, N. Ohno, Computational multiple scattering analysis for shear wave propagation in unidirectional composites, Int. J. Solids Struct. 41 (2004), 435-457.

[26] F. Kobayashi, S. Biwa, N. Ohno, Wave transmission characteristics in periodic media of finite length: multilayers and fiber arrays, Int. J. Solids Struct. 41 (2004), 7361-7375.

[27] S. Biwa, F. Kobayashi, N. Ohno, Influence of disordered fiber arrangement on SH wave transmission in unidirectional composites, Mech. Mater. 39 (2007), 1-10.

[28] S. Biwa, T. Kamiya, N. Ohno, Multiple scattering simulation of ultrasonic shear wave in unidirectional carbon/epoxy composites, Mater. Trans. 48 (2007), 1196-1201.

[29] A. Lange, A. Harker, N. Saffari, A multilevel multipole method for modeling elastic-wave multiple scattering in fiber-reinforced composites, Review of Progress in Quantitative Nondestructive Evaluation 22 (2003), 85-92.

[30] T. Saitoh, S. Hirose, T. Fukui, Application of fast multipole boundary element method to multiple scattering analysis of acoustic and elastic waves, Review of Quantitative Nondestructive Evaluation 26 (2007), 79-86.

[31] K. Nakashima, S. Biwa, E. Matsumoto, Elastic wave transmission and stop band characteristics in unidirectional composites, J. Solid Mech. Mater. Eng. 2 (2008), 1195-1206.

[32] J. L. Rose, Ultrasonic Waves in Solid Media, Cambridge University Press, Cambridge, 1999.

[33] Y.-H. Pao, C.-C. Mow, Diffraction of Elastic Waves and Dynamic Stress Concentrations, Crane Russak, New York, 1971.

[34] S. I. Rokhlin, W. Huang, Y. C. Chu, Ultrasonic scattering and velocity methods for characterization of fibre-matrix interphases, Ultrasonics 33 (1995), 351-364.

[35] J. Feder, Random sequential adsorption, J. Theo. Biol. 87 (1980), 237-254. 